\title{
RELAÇÃO DA POSTURA CERVICAL E OCLUSÃO DENTÁRIA EM CRIANÇAS RESPIRADORAS ORAIS
}

\author{
Relationship between cervical posture and dental occlusion \\ in mouth-breathing children
}

\author{
Lara Jansiski Motta ${ }^{(1)}$, Manoela Domingues Martins ${ }^{(2)}$, \\ Kristianne Porta Santos Fernandes ${ }^{(3)}$, Raquel Agnelli Mesquita-Ferrari ${ }^{(4)}$, \\ Daniela Aparecida Biasotto-Gonzalez ${ }^{(5)}$, Sandra Kalil Bussadori ${ }^{(6)}$
}

\section{RESUMO}

Objetivo: avaliar e comparar a postura de cabeça e pescoço e a relação com a classe oclusal, entre crianças respiradoras orais e respiradoras nasais. Métodos: realizou-se um estudo observacional, de corte transversal em que participaram 110 crianças divididas em Grupo I: respiradoras nasais e Grupo II: respiradoras orais. Para avaliação da respiração utilizou-se o teste do vapor no espelho e água na boca, e exame clínico intra-oral para classificação oclusal de Angle. A análise da postura foi realizada por meio de fotogrametria e o software Alcimage ${ }^{\circledR}$ para mensuração do ângulo pré-definido pelos pontos Processo Espinhoso da sétima vértebra cervical, Manúbrio do Esterno e Ápice do Mento. Os dados obtidos foram tabulados e submetidos à análise estatística, utilizando o programa SPSS 12.0, num intervalo de confiança de 95\%. Resultados: foi possível observar valores significativamente maiores do ângulo cervical para o Grupo II $\left(96,59^{\circ} \pm 8,79\right)$ quando comparado ao Grupo I $\left(86,60^{\circ} \pm 8,53\right)(p<0,001)$. As crianças respiradoras orais apresentaram oclusão classe II de Angle $(p<0,05)$. Os participantes classe II apresentaram maior ângulo cervical, diferindo estatisticamente dos pacientes classe I $(p<0,05)$. Conclusão: existe relação entre respiração oral, alteração na postura da cabeça e pescoço e oclusão classe II de Angle nas crianças estudadas, sendo a anteriorização da cabeça a alteração mais evidente em crianças respiradoras orais.

DESCRITORES: Respiração Bucal; Coluna Vertebral; Oclusão Dentária

(1) Cirurgiã-Dentista; Professora da disciplina de Odontopediatria da Faculdade de Odontologia da Universidade Nove de Julho, UNINOVE, São Paulo, SP; Mestre em Ciências da Reabilitação pela Universidade Nove de Julho.

(2) Cirurgiã-Dentista; Professora Doutora do Programa de Mestrado em Ciências da Reabilitação da Universidade Nove de Julho, UNINOVE, São Paulo, SP.

(3) Cirurgiã-Dentista; Professora Doutora do Programa de Mestrado em Ciências da Reabilitação da Universidade Nove de Julho, UNINOVE, São Paulo, SP.

(4) Fisioterapeuta; Professora Doutora do Programa de Mestrado em Ciências da Reabilitação da Universidade Nove de Julho, UNINOVE, São Paulo, SP.

(5) Fisioterapeuta; Professora Doutora do Programa de Mestrado em Ciências da Reabilitação da Universidade Nove de Julho, UNINOVE, São Paulo, SP.

(6) Cirurgiã-Dentista; Professora Doutora do Programa de Mestrado em Ciências da Reabilitação da Universidade Nove de Julho, UNINOVE, São Paulo, SP.

Conflito de interesses: inexistente

\section{INTRODUÇÃO}

O respirador oral é aquele indivíduo que respira predominantemente pela boca, a partir de qualquer idade, independentemente da causa. Nas crianças, as causas mais frequentes incluem a hipertrofia de amídalas palatinas; a presença de pólipos nasais; as rinites de uma forma geral; a rinosinusite; a presença de cavidade nasal estreita; a atresia congênita de coanas ou, simplesmente, por hábito ${ }^{1-3}$. Com o tempo, a respiração oral crônica pode levar a alterações no crescimento e no desenvolvimento dentofaciais, principalmente nos primeiros anos de vida, quando se tem uma grande velocidade de crescimento das estruturas faciais e do crânio ${ }^{3}$.

A respiração oral pode acarretar prejuízos em diversas áreas ${ }^{4-7}$, levando os indivíduos a apresentarem características como: alterações 
craniofaciais, da postura corporal, da musculatura facial, da oclusão, das funções de mastigação e deglutição, distúrbios do sono, da concentração e atenção e, ainda, incidência aumentada de episódios de otites e outras patologias da orelha média, as quais determinam perdas auditivas. A persistência da alteração das vias aéreas superiores determina um prejuízo na mecânica ventilatória, com desequilíbrio das forças musculares que podem produzir disfunções temporomandibulares, torácicas e, consequentemente, desvios em todos os eixos posturais ${ }^{4,8-10}$.

Enlow ${ }^{11}$ cita mudanças na postura do respirador oral, como: abaixamento da mandíbula, posicionamento da língua para baixo e para frente e inclinação da cabeça para trás. Em decorrência dessas modificações haveria uma alteração de toda a postura corporal. Uma descrição mais detalhada das alterações posturais do respirador oral foi feita por Aragão ${ }^{12}$ que explica que a criança respiradora oral, para conseguir respirar melhor, coloca o pescoço para frente, retificando o trajeto das vias respiratórias e fazendo o ar chegar mais rápido da boca aos pulmões. Ao anteriorizar a cabeça e pescoço, as escápulas se elevam e a região anterior do tórax fica deprimida, tornando a respiração mais rápida e curta, com pequena ação do diafragma 11-13.

A relação entre respiração oral e alterações dentomaxilares, como protrusão e apinhamento dentário, más oclusões dentárias, gengivites, cáries dentárias e palato ogival é amplamente descrita na literatura 1-3,5,7. As opiniões, em relação à causalidade, ainda são controversas quanto à má oclusão dentária e o paciente respirador oral. De acordo com alguns autores, a respiração oral completa não provocaria má oclusão, pois a musculatura se encontraria flácida, não funcional e, por consequência, não lesiva. Ao contrário, a respiração oral parcial provocaria a má oclusão dentária, por necessitar de ação muscular potente, de forma anormal ${ }^{14,15}$. Por outro lado, autores como Aragão ${ }^{12}$ e Breuer ${ }^{16}$ afirmam que cerca de $90 \%$ dos pacientes respiradores orais apresentam má oclusão dentária.

Referente às alterações oclusais, estudos descrevem forte relação entre respiração oral e oclusão Classe II de Angle 1,3,5,7,14,17-19.

Diante das alterações posturais e desequilíbrios musculares decorrentes da respiração oral descritos na literatura, observa-se a necessidade da avaliação multidisciplinar destes pacientes. Sendo assim, o objetivo deste trabalho foi avaliar e comparar a postura de cabeça e pescoço e a relação com a classe oclusal, entre crianças respiradoras orais e respiradoras nasais.

\section{MÉTODOS}

Todos os responsáveis receberam informações sobre o projeto e assinaram termo de consentimento formal, de acordo com a resolução 196/96 do Conselho Nacional de Saúde, autorizando publicação dos dados e imagens.

Desenvolveu-se um estudo observacional, de corte transversal, para avaliação das funções do sistema estomatognático e da postura da cabeça, dos alunos de uma escola municipal no interior de São Paulo. Dos 463 alunos matriculados, cinco não concordaram em participar e quatro não estavam comparecendo às aulas há 20 dias ou mais, portanto foram avaliados 454 alunos. Para o recrutamento e formação dos grupos de estudo, utilizouse como critérios de inclusão a faixa etária ( 6 a 10 anos) e a presença dos primeiros molares permanentes e foram excluídas do estudo crianças em tratamento médico do trato respiratório, com má formação crânio-facial. Os alunos que contemplaram os critérios de inclusão $(n=110)$ foram avaliados quanto ao padrão respiratório e divididos em dois grupos: Grupo I - Crianças respiradoras nasais e Grupo II- Crianças respiradoras orais.

O tipo de respiração de cada participante foi determinado por meio de uma avaliação clínica e pela realização de testes específicos (teste do espeIho e de água na boca), confirmados pela avaliação de um otorrinolaringologista. Na avaliação clínica foi verificada a presença ou ausência dos seguintes sinais: face alongada, olhos caídos, olheiras, lábio superior estreito (fino), lábios ressecados, lábios hipotônicos, lábio inferior invertido, narinas estreitas, palato ogival, selamento labial inadequado e mordida aberta anterior. Todos os procedimentos foram realizados na própria escola.

O teste do espelho constou da colocação de um espelho refletor abaixo das narinas da criança e verificação da formação de vapor, decorrente da respiração, na parte inferior ou superior do mesmo. $O$ vapor na parte superior do espelho indicou a respiração nasal e na parte inferior ou ambas as partes do espelho considerou-se como respiração nasal.

O teste de água na boca consistiu na colocação de um pouco de água na boca da criança e manutenção dos lábios em contato, sem engolir a água por 3 minutos cronometrados, observando através da comissura labial, se houve esforço no decorrer do tempo. As crianças que não conseguiram permanecer por 3 minutos com os lábios em contato foram consideradas respiradoras orais ${ }^{20}$.

Após o exame, 56 crianças foram consideradas respiradoras nasais, compondo o grupo I; e 54 crianças com padrão de respiração oral constituíram o grupo II. O Grupo I foi composto por 
27 meninos e 29 meninas, enquanto o Grupo II foi constituído por 30 meninos e 24 meninas.

Em seguida, as crianças foram submetidas a exame clínico intra-oral, sendo classificadas de acordo com a classe oclusal de Angle, obtida pela análise da relação dos primeiros molares permanentes. Os portadores de classe I possuem um perfil facial reto e bom posicionamento da maxila e mandíbula, a cúspide mesio-vestibular do primeiro molar superior oclui no sulco mesio-vestibular do primeiro molar inferior. As más oclusões da classe II caracterizam-se pelo fato de o primeiro molar inferior situar-se distalmente ao primeiro molar superior, sendo também denominada distoclusão. A má oclusão classe III é caracterizada pela mesioclusão do primeiro molar inferior, ou seja, a cúspide mésiovestibular do primeiro molar superior, oclui posteriormente ao sulco mesio-vestibular do primeiro molar inferior ${ }^{1}$.

Todos os participantes foram fotografados para avaliação postural de cabeça e pescoço, a análise foi realizada pelo software Alcimagem $® 2.1$, sendo que este oferece uma análise quantitativa dos ângulos da imagem por meio de pontos pré-determinados (marcadores). As fotos foram realizadas com os voluntários em pé, vista lateral, perfil direito, posicionados em uma marcação pré-estabelecida no chão. A máquina fotográfica (Kodak ${ }^{\mathrm{TM}} \mathrm{Z740}$ 7.1) foi fixada a um metro e meio do participante sobre uma base fixa com altura e zoom ajustáveis para a retirada da fotografia. Os participantes permaneceram com o tronco completamente despido, ou regata no caso do gênero feminino, para que os pontos analisados ficassem claramente visíveis.

Os pontos analisados no software foram: Processo Espinhoso da $7^{a}$ Vértebra Cervical, Manúbrio do Esterno e Ápice do Mento. Portanto, o ângulo estabelecido para análise a partir destes três pontos de referência foi manúbrio do esterno segundo Rocabado ${ }^{21}$, no qual foram observadas variações da postura da cabeça em repouso (Figura 1).

Este estudo foi aprovado pelo Comitê de Ética em Pesquisa Humana sob o no 82622/08.

Para a análise estatística foi utilizado o teste Qui-quadrado ( $\chi 2$ ), para avaliar a associação entre variáveis qualitativas. Para comparação das diferenças de postura (médias dos ângulos) entre o grupo respirador oral e o grupo de respiradores nasais, foi utilizado o Teste t de Student, com nível de significância estabelecido em $5 \%(p<0,05)$. Para aplicação dos testes estatísticos foi utilizado o Programa Estatístico SPSS 12.0.

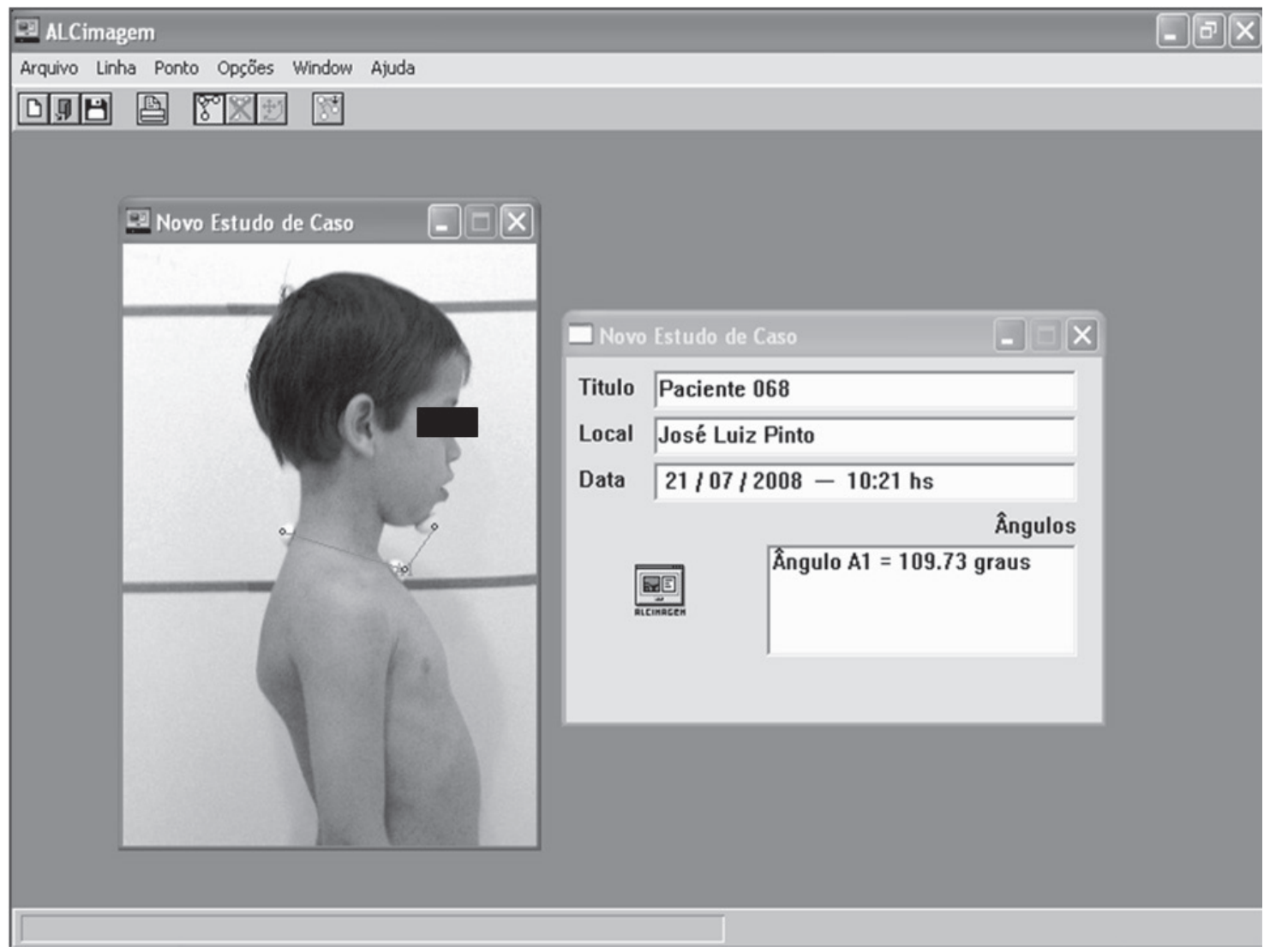

Figura 1 - Análise Postural 


\section{RESULTADOS}

A média do ângulo mensurado foi estatisticamente significante maior para o Grupo II $\left(96,59^{\circ} \pm 8,79\right)$ quando comparado ao Grupo I $\left(86,60^{\circ} \pm 8,53\right)(p<0,001)$.

A avaliação da classe oclusal de Angle mostrou que a maioria das crianças do Grupo I $(55,4 \%)$ era portadora de oclusão classe I, enquanto que a maioria das crianças do Grupo II (68,5\%) era classe II (Tabela 1). A relação entre Classe II de Angle e padrão respiratório oral mostrou-se estatisticamente significante $(p<0,05)$.

Quando avaliado o ângulo cervical em relação às classes oclusais (Tabela 2), observou-se diferença estatisticamente significante entre os indivíduos classe II, com valor médio do ângulo de $93,16^{\circ}$, e classe I, com média de $88,37^{\circ}$. Os participantes portadores de classe III, com média $94,97^{\circ}$ não diferiram estatisticamente dos demais $(p>0,05)$.

$\mathrm{Na}$ análise da diferença de médias dos ângulos cervicais, nas diferentes classes oclusais em relação ao padrão respiratório, observou-se diferença estatisticamente significante (Tabela 3 ) nas três classes oclusais $(p<0,05)$.

\section{DISCUSSÃO}

No presente estudo foi possível observar correlação entre respiração oral e alteração postural da cabeça e pescoço em crianças nas diferentes

Tabela 1 - Distribuição das crianças em relação ao padrão respiratório e classe oclusal

\begin{tabular}{|c|c|c|c|c|c|}
\hline \multirow{2}{*}{ Padrão Respiratório } & & \multicolumn{3}{|c|}{ Classe Oclusal } & \multirow{2}{*}{ Total } \\
\hline & & I & II & III & \\
\hline Grupo I & $\mathrm{N}$ & $31(55,4 \%)$ & $20(35,7 \%)$ & $5(8,9 \%)$ & 56 (100\%) \\
\hline Grupo II & $\mathrm{N}$ & $10(18,5 \%)$ & $37(68,5 \%)$ & $7(13,0 \%)$ & $54(100 \%)$ \\
\hline Total & $\mathrm{N}$ & $41(37,3 \%)$ & $57(51,8 \%)$ & $12(10,9 \%)$ & $110(100 \%)$ \\
\hline
\end{tabular}

Tabela 2 - Diferença de médias dos ângulos cervicais em relação à classe oclusal

\begin{tabular}{cccccc}
\hline $\begin{array}{c}\text { Classe } \\
\text { Oclusal }\end{array}$ & $\begin{array}{c}\text { Classe } \\
\text { Oclusal }\end{array}$ & $\begin{array}{c}\text { Diferença de } \\
\text { Médias }\end{array}$ & $\mathbf{p}$ & \multicolumn{2}{c}{ Intervalo de Confiança (95\%) } \\
\hline I & II & $-4,78678\left(^{*}\right)$ & 0,018 & $-8,7490$ &,- 8245 \\
& III & $-6,00248$ & 0,064 & $-12,3531$ &, 3481 \\
II & I & $4,78678\left(^{*}\right)$ & 0,018 &, 8245 & 8,7490 \\
& III & $-1,21570$ & 0,696 & $-7,3612$ & 4,9298 \\
III & I & 6,00248 & 0,064 &,- 3481 & 12,3531 \\
& II & 1,21570 & 0,696 & $-4,9298$ & 7,3612 \\
\hline
\end{tabular}

*Diferença de médias significante $p<0,05$

Tabela 3 - Ângulos Cervicais dos Grupos I e II em relação à classe oclusal

\begin{tabular}{ccccc}
\hline & & $\begin{array}{c}\text { Média dos ângulos } \\
\text { cervicais }\end{array}$ & Desvio Padrão & p \\
\hline \multirow{2}{*}{ Classe I } & Grupo I & 85,04 & 8,63 & $0,000^{*}$ \\
& Grupo II & 98,68 & 8,05 & \\
Classe II & Grupo I & 88,93 & 7,39 & $0,000^{*}$ \\
& Grupo II & 95,44 & 9,53 & \\
Classe III & Grupo I & 86,95 & 11,72 & $0,002^{*}$ \\
\hline
\end{tabular}

*Diferença de médias significante $p<0,05$ 
classes oclusais de Angle. As crianças com respiração oral mudam a postura da cabeça, provavelmente, para adaptar a angulação da faringe para facilitar a entrada de ar pela boca, na tentativa de obter um melhor fluxo aéreo superior ${ }^{1,11,13}$. A mudança do eixo da cabeça altera também a posição de repouso mandibular, os contatos oclusais, os planos óticos e bipupilar. A estas mudanças, podem seguir-se movimentos adaptativos do corpo em busca de uma postura mais confortável e de equilíbrio ${ }^{1,4,22}$. Portanto, a criança que possui respiração oral, altera o funcionamento fisiológico da respiração, e a postura da cabeça acaba por sofrer transformações. Como consequência, as relações da cabeça com o pescoço e deste com o tronco, também poderão estar alteradas. Os resultados do presente estudo permitiram verificar um aumento do ângulo cervical nas crianças respiradoras orais em comparação com as respiradoras nasais, 0 que caracteriza uma posição mais anteriorizada da cabeça.

No respirador oral, ocorre um desvio funcional da musculatura oral, do rosto e da postura, que levam a um desenvolvimento inadequado do aparelho mastigatório, o que é uma indicação para a má oclusão 2,3,13,22-24.

Neste estudo, os resultados demonstraram que as crianças respiradoras orais eram na maioria $(68,5 \%)$ portadores de má oclusão classe II de Angle. Estes resultados podem ser justificados pela afirmação de que a Classe II é o tipo de má oclusão acompanhada e, pelo menos, no início, agravada, se não causada, pela respiração oral devido a alguma forma de obstrução nasal ${ }^{23,25}$.

Quando avaliada a postura da cabeça em relação às classes oclusais, na presente pesquisa, os participantes portadores de má oclusão classe II apresentaram uma posição mais anteriorizada da cabeça, diferindo estatisticamente dos participantes portadores de classe I, confirmando a afirmação que crianças com má oclusão classe I mantêm a posição da cabeça em equilíbrio; as com classe II alteram a posição da cabeça e dos ombros para frente ${ }^{1,26}$.

A grande maioria dos estudos de correlação entre respiração oral, classe oclusal e postura da cabeça e pescoço é realizada por meio de análises cefalométricas ou exame clínico 1,3,7,13,14,17. Neste estudo optou-se pela fotogrametria. A análise fotográfica é um método acessível, sem os custos de equipamentos sofisticados de análise de movimento ou possíveis riscos de repetidas avaliações radiológicas ${ }^{4}$. O software ALCimagem $®$, que executa uma análise angular em imagens fotográficas, permite avaliar quantitativamente os desvios posturais, além de evitar a subjetividade do examinador ${ }^{27}$. O estudo de Lima et al ${ }^{28}$ demonstrou a confiabilidade deste programa por meio de medidas angulares utilizadas na avaliação biofotogramétrica de crianças respiradoras nasais e orais.

Os dados obtidos alertam para a discussão da avaliação multidisciplinar do respirador oral precocemente, pois, tanto a condição de respirador oral quanto a da alteração postural da cabeça, podem comprometer o desenvolvimento adequado do complexo crânio-facial.

\section{CONCLUSÃO}

Os resultados obtidos permitiram concluir que existe relação entre respiração oral, a postura da cabeça e pescoço e oclusão classe II de Angle nas crianças estudadas. Em sujeitos com respiração oral, a anteriorização da cabeça foi predominante. 


\section{ABSTRACT}

Purpose: to evaluate and compare head and neck posture, and the relationship between occlusal class, among mouth and nasal breathing children. Methods: an observational, cross-sectional study was carried out, in which 110 children took part. These were divided into Group I: nasal breathers, and Group II: mouth breathers. For evaluating the respiration, we used mirror clouding and filling the mouth with water tests, with intrabuccal clinical exam for the Angle occlusal classification. Posture analysis was carried out by means of photogrammetry, and the software Alcimagem $®$ was used to measure the predefined angle by the protuberances of the Spinous Process of the $7^{\text {th }}$ Cervical Vertebra, Manubrium of the sternum and Mentum Vertex. The obtained data were tabulated and submitted to a statistical analysis, using the program SPSS 12.0 with a 95\% confidence interval. Results: a larger cervical angle was observed for Group II (96.59 $\left.{ }^{\circ} \pm 8.79\right)$ than for Group I $\left(86.60^{\circ} \pm 8.53\right)$, showing statistically significant differences $(p<0.001)$. The majority $(68.5 \%)$ of the mouth breathing individuals presented class II occlusion Angle, and this relationship was significant $(p<0.05)$. The class II individuals showed a larger cervical angle, differing statistically from the class I patients $(p<0.05)$. Conclusion: it may be concluded that there is a connection between mouth breathing, alteration in head and neck posture and class II occlusal Angle in the studied children. In mouth breathing subjects, forward inclination of the head is predominant.

KEYWORDS: Mouth Breathing; Spine; Dental Occlusion

\section{REFERÊNCIAS}

1. Costa JR, Pereira SRA, Mittri G, Motta JC, Pignatari SS, Weckx LLM. Relação da oclusão dentária com a postura de cabeça e coluna cervical em crianças respiradoras orais. Rev Paul Pediatr. 2005; 23(2):88-93.

2. Abreu RR, Rocha RL, Lamounier JA, Guerra AFM. Etiologia, manifestações clínicas e alterações presentes nas crianças respiradoras orais. J Pediatr. 2008; 84(6):529-35.

3. Ianni Filho D, Bertolini MM, Lopes ML. Contribuição multidisciplinar no diagnóstico e no tratamento das obstruções da nasofaringe e da respiração bucal. Rev Clin Ortodon Dental Press. 2006; 4(6):90-102.

4. Nouer DF, Souza MA, Romano FL, Coelho-Ferraz MJP. Fatores etiológicos da respiração bucal. In: Coelho-Ferraz, organizadora. Respirador bucal: uma visão multidisciplinar. São Paulo: Lovise; 2005. p.43-50.

5. Oliveira CF, Busanello AR, Silva AMT. Ocorrência de má oclusão e distúrbio articulatório em crianças respiradoras orais de escolas públicas de Santa Maria, Rio Grande do Sul. RGO. 2008; 56(2):169-74. 6. Lessa FCR, Enoki C, Feres MFN, Valcra FCP, Lima WTA, Matsumoto MAN. Influência do padrão respiratório na morfologia craniofacial. Rev Bras Otorrinolaringol. 2005; 71(2):156-60.

7. Bicalho GP, Motta AR, Vicente LCC. Avaliação da deglutição em crianças respiradoras orais. Rev. CEFAC. 2006; 8(1):50-5.
8. Chaves TC, Grossi DB, Oliveira AS, Bertolli F, Holtz A, Costa D. Correlation between signs of temporomandibular (TMD) and cervical spine (CSD) disorders in asthmatic children. J Clin Pediatr Dent. 2005; 29(4):287-92.

9. Frasson JMD, Magnani MBBA, Nouer DV, Siqueira VCV, Lunardi N. Estudo cefalométrico comparativo entre respiradores nasais e predominantemente bucais. Rev Bras Otorrinolaringol. 2006; 72(1):72-82. 10. Marins RS. Síndrome do respirador bucal e modificações posturais em crianças e adolescentes: a importância da fisioterapia na equipe multidisciplinar e interdisciplinar. Rev Fisioter Mov. 2001; 14(1):45-52.

11. Enlow DH. Crescimento facial. São Paulo: Artes Médicas; 1993. 553p.

12. Aragão W. Respirador bucal. J Pediatr. 1988; 64(8):349-52.

13. Cuccia AM, Lotti M, Caradonna D. Oral breathing and head posture. Angle Orthod. 2007; 78(1):77-82. 14. Rejman $R$, Martins DR, Scavone Júnior $H$, Cotrim-Ferreira FA, Vellini-Ferreira F. Estudo comparativo das dimensões transversais dos arcos dentários entre jovens com oclusão normal e má oclusão de Classe II, $1^{a}$ divisão. Dental Press Ortodon Ortop Facial. 2006; 11(4):118-25.

15. Andrade FV, Andrade DV, Araújo AS, Ribeiro ACC, Deccax LDG, Nemr K. Alterações estruturais de órgãos fonoarticulatórios e más oclusões dentárias em respiradores orais de 6 A 10 Anos. Rev. CEFAC. 2005; 7(3):318-25. 
16. Breuer J. El paciente respirador bucal. Rev Assoc Odontol Argent. 1989; 77(3):102-6.

17. Peltomäki T. The effect of mode of breathing on craniofacial growth-revisited. Eur J Orthod. 2007; 29(5):426-9.

18. Pirilä-Parkkinen K, Pirttiniemi $P$, Nieminen $P$, Tolonen U, Pelttari U, Löppönen $H$. Dental arch morphology in children with sleep-disordered breathing. Eur J Orthod. 2009; 31(2):160-7.

19. Clark WD. Preventing dentofacial abnormalities with the proper correction of pediatric upper airway obstruction. Arch Otolaryngol Head Neck Surg. 2005; 131(10):916-8.

20. Jorge EP, Abrão J, Castro ABBAT. Estudo da resistência nasal em pacientes com má oclusão de classe II divisão $1^{\underline{a}}$ de Angle, utilizando a rinomanometria anterior ativa. Rev Dental Press Ortodon Ortop Max. 2001; 6(1):15-30.

21. Rocabado Seaton M. Relaciones biomecánicas de las regiones craneales, cervicales e hioideas. Ortodoncia. 1994; 58(115):51-6.

22. Neiva PD, Kirkwood RN. Mensuração da amplitude de movimento cervical em crianças respiradoras orais. Rev Bras Fisioter. 2007; 11(5):355-60.
25. Sies ML, Farias SR, Vieira MM. Respiração oral: relação entre o tipo facial e a oclusão dentária em adolescentes. Rev Soc Bras Fonoaudiol. 2007; 12(3):191-8.

24. Solow B, Sandham A. Cranio-cervical posture: a factor in the development and function of the dentofacial structures. Eur J Orthod. 2002 Oct; 24(5):447-56.

25. Almeida FL, Silva AMT, Serpa EO. Relação entre má oclusão e hábitos orais em respiradores orais. Rev. CEFAC. 2009; 11(1):86-93. dx.doi. org/10.1590/S1516-18462009005000005

26. Bricot B. O captor dento-oclusal. In: Bricot B, organizador. Posturologia. São Paulo: Ícone; 1999. p. 159-91.

27. Baraúna MA, Ricieri D. Biofotogrametria: recurso diagnóstico do fisioterapeuta. Rev Coffito. 2002; 17:8-11.

28. Cattoni DM, Fernandes FDM, Di Francesco RC, Latorre MRDO. Medidas e proporções antropométricas orofaciais de crianças respiradoras orais. Rev Soc Bras Fonoaudiol. 2008; 13(2):119-26.

RECEBIDO EM: 06/03/2009

ACEITO EM: 18/08/2009

Endereço para correspondência:

Lara Jansiski Motta

Av. Getúlio Vargas, 386 ap. 53

São Roque - SP

CEP: $18130-430$

E-mail: larajmotta@terra.com.br 